\title{
Bone mineral content in cystic fibrosis patients: correlation with fat-free mass
}

Françoise Salamoni, Michel Roulet, François Gudinchet, Marianne Pilet, Daniel Thiébaud, Peter Burckhardt

\begin{abstract}
Objective-To assess the bone mineral content in well nourished patients with cystic fibrosis and to seek a correlation with fat-free mass.

Methods-Fourteen cystic fibrosis patients aged 6 to 20 years were studied and compared to 14 healthy controls matched for gender, age, and nutritional status. Bone mineral content was determined by dual energy $x$ ray absorptiometry (DEXA).

Results-Nutritional inquiry showed higher ingestion of macronutrients and micronutrients by cystic fibrosis patients than by controls. Mean whole skeleton bone mineral content was $1 \cdot 184$ (SD 0.536) $\mathrm{kg}$ in cystic fibrosis patients and 1.229 $(0 \cdot 576) \mathrm{kg}$ in controls $(p=0 \cdot 84)$. Mean lumbar spine bone mineral content was $0.031(0.013) \mathrm{kg}$ and $0.031(0.016) \mathrm{kg}$, respectively $(p=0.99)$. Anthropometry, bioelectrical impedance analysis, and DEXA showed that fat-free mass was similar in the two groups. Bone mineral content was strongly correlated to fat-free mass. Mean blood calcium, phosphorus, serum 25-hydroxyvitamin D (25-OHD), parathyroid hormone (PTH), and osteocalcin were similar in both groups.

Conclusions-Bone mineral content and body composition are normal in a well nourished young cystic fibrosis population. Osteopenia previously reported in cystic fibrosis patients probably has nutritional origins and is therefore not related to a primary defect in bone mineral metabolism.
\end{abstract}

(Arch Dis Child 1996; 74: 314-318)

University Hospital, Lausanne, Switzerland:

Nutrition Unit, Department of Paediatrics F Salamoni M Roulet

M Pilet

Department of Radiology F Gudinchet

Department of Internal Medicine D Thiébaud P Burckhardt

Correspondence to: Dr M Roulet, Nutrition Unit, Department of Paediatrics, University Hospital, CHUV, CH-1011 Lausanne, Switzerland.

Accepted 16 January 1996 um-vitamin D metabolic disorder remair unclear. It might also be due to lack of weight bearing physical activity brought on by general deterioration in health in cystic fibrosis have been reported in cystic fibro patients. ${ }^{4-6}$ Whether this is a result of protein-
The prognosis of cystic fibrosis patients has mean survival time is now 30 years, ${ }^{1}$ the prognosis. Body composition in cystic fibrosis patients differs from that in healthy controls ${ }^{2} 3$ patients, especially in adults. In healthy subjects, peak bone mass is reached at the end of the growth spurt ${ }^{78}$ and bone mineral content in adulthood depends more on peak bone mass than on subsequent mineral loss. ${ }^{9}$ As cystic fibrosis patients enjoy longer lives, assessment of their bone mineralisation during childhood and adolescence is important for the eventual prevention of osteopenia.

In this study we investigated whether cystic fibrosis patients have a primary defect in bone mineral and vitamin $\mathrm{D}$ metabolism independent of protein-energy malnutrition. We also examined the relations between the bone mineral content, fat-free mass, and indices of bone mineralisation in well nourished patients with cystic fibrosis.

\section{Methods}

SUBJECTS

Fourteen well nourished (weight for height ratio 0.85 or above) cystic fibrosis patients (four females, 10 males), 6.6 to 19.9 years old, were recruited from our cystic fibrosis outpatient clinic. All but two received enteric coated microspheres of pancreatic enzymes and multivitamin supplements providing at least $500 \mathrm{IU}$ of vitamin D daily. Seven were on a polymeric liquid formula as a dietary supplement. The most common cystic fibrosis mutations (DF508, 3905insT, G542X, R553X, G551D, 1717-1G-A) were looked for. The severity of cystic fibrosis disease was evaluated by the Shwachman-Kulczycki score (possible scores range from 0 to 100 , the lowest being the worst), ${ }^{10}$ and pulmonary $x$ ray lesions by the Chrispin-Norman score (possible scores range from 0 to 38 , the highest being the worst). ${ }^{11}$ Pubertal stage was determined using the Tanner score. ${ }^{12}$ Patients with clinical heart failure or oedema, and those receiving insulin, corticosteroids, thiazides, anticonvulsants, and other drugs affecting bone were excluded.

Fourteen healthy subjects were recruited as controls by cystic fibrosis patients among their schoolmates or friends. They were matched one to one for gender, age, and height. They underwent exactly the same procedure as cystic fibrosis patients except that no chest radiography was done.

Energy, protein, calcium, phosphorus, and vitamin $D$ intakes were estimated by a 72 hour recall. ${ }^{13-15}$

All cystic fibrosis patients and controls lived in Switzerland (latitude of $46^{\circ}$ north), a country with a mean of 1800 hours of sunlight per year. 
Informed consent was obtained from each subject and from parents when the subject was a minor. The study was approved by the ethics committee of the Faculty of Medicine of Lausanne. The procedures followed were in accordance with the Helsinski Declaration of 1975 , as revised in 1983.

\section{BODY COMPOSITION AND BONE MINERAL} CONTENT

Weight and height were measured using standard techniques. Weight for height ratio, which is the ratio between the actual weight of the studied subject and the theoretical weight of an 'ideal' person of the same height, was calculated. ${ }^{16}$ Fat-free mass was assessed by anthropometry, ${ }^{1617}$ bioelectrical impedance analysis, ${ }^{18-20}$ and dual energy $x$ ray absorptiometry (Hologic QDR 2000). ${ }^{21}$ Bone age was determined according to Greulich and Pyle. ${ }^{22}$ Bone mineral content of the whole skeleton and of the lumbar spine (L1-L4) were evaluated by DEXA.

\section{LABORATORY DETERMINATIONS}

Plasma calcium and phosphorus, and urinary calcium, creatinine, and hydroxyproline were measured by standard methods. Serum 25OHD was determined by a protein binding assay (25-hydroxyvitamin $D \quad\left[{ }^{3} \mathrm{H}\right]$ assay system, code TRK 860, Amersham International), serum $\mathrm{PTH}$ by a radioisotopic assay (INTACT PTH parathyroid hormone 100T-kit; catalogue No 40-2170, Nichols Institute), and serum osteocalcin by immunoradiometric assay (ELSA-OSTEO, CIS Bio International). Molar calcium/creatinine and hydroxyproline/creatinine ratios were calculated in the morning urine.

\section{STATISTICAL ANALYSIS}

After a test for normality of distribution, unpaired $t$ tests and Mann-Whitney $U$ tests were used to compare the two groups of

Table 1 Gender, age, bone age, pubertal stage, and anthropometric characteristics of subjects (cystic fibrosis/control)

\begin{tabular}{|c|c|c|c|c|c|c|c|}
\hline Pair & Gender & $\begin{array}{l}\text { Age } \\
\text { (years) }\end{array}$ & $\begin{array}{l}\text { Bone age } \\
\text { (years) }\end{array}$ & $\begin{array}{l}\text { Pubertal } \\
\text { stage }^{\star}\end{array}$ & $\begin{array}{l}\text { Weight } \\
(\mathrm{kg})\end{array}$ & $\begin{array}{l}\text { Height } \\
\text { (m) }\end{array}$ & $\begin{array}{l}\text { Weight for } \\
\text { height ratio† }\end{array}$ \\
\hline $\begin{array}{r}1 \\
2 \\
3 \\
4 \\
5 \\
6 \\
7 \\
8 \\
9 \\
10 \\
11 \\
12 \\
13 \\
14\end{array}$ & $\begin{array}{l}M / M \\
M / M \\
M / M \\
M / M \\
F / F \\
M / M \\
M / M \\
F / F \\
F / F \\
F / F \\
M / M \\
F / F \\
M / M \\
M / M\end{array}$ & $\begin{array}{c}9 \cdot 1 / 9 \cdot 8 \\
11 \cdot 1 / 10 \cdot 2 \\
6 \cdot 6 / 7 \cdot 4 \\
19 \cdot 9 / 20 \cdot 5 \\
17 \cdot 3 / 17 \cdot 0 \\
16 \cdot 5 / 16 \cdot 8 \\
11 \cdot 6 / 11 \cdot 3 \\
11 \cdot 9 / 11 \cdot 6 \\
11 \cdot 8 / 11 \cdot 9 \\
12 \cdot 8 / 12 \cdot 5 \\
10 \cdot 6 / 10 \cdot 5 \\
9 \cdot 4 / 10 \cdot 6 \\
11 \cdot 5 / 11 \cdot 3 \\
10 \cdot 2 / 10 \cdot 3\end{array}$ & $\begin{array}{c}12 \cdot 5 / 10 \cdot 0 \\
9 \cdot 0 / 8 \cdot 5 \\
5 \cdot 0 / 4 \cdot 5 \\
18 \cdot 0 / 20 \cdot 5 \\
17 \cdot 0 / 17 \cdot 0 \\
16 \cdot 0 / 17 \cdot 0 \\
11 \cdot 0 / 11 \cdot 0 \\
10 \cdot 0 / \mathrm{ND} \\
12 \cdot 0 / 11 \cdot 0 \\
11 \cdot 0 / 12 \cdot 0 \\
9 \cdot 0 / 10 \cdot 0 \\
9 \cdot 0 / 10 \cdot 0 \\
12 \cdot 5 / 11 \cdot 0 \\
9 \cdot 0 / 9 \cdot 0\end{array}$ & $\begin{array}{l}\text { P3/P1 } \\
\text { P1/P1 } \\
\text { P1/P1 } \\
\text { P5/P5 } \\
\text { P5/P5 } \\
\text { P4/P4 } \\
\text { P1/P1 } \\
\text { P2/P2 } \\
\text { P3/P2 } \\
\text { P3/P3 } \\
\text { P1/P1 } \\
\text { P1/P2 } \\
\text { P1/P1 } \\
\text { P1/P1 }\end{array}$ & $\begin{array}{l}30 \cdot 4 / 26 \cdot 4 \\
29 \cdot 0 / 28 \cdot 9 \\
21 \cdot 8 / 19 \cdot 0 \\
60 \cdot 8 / 56 \cdot 9 \\
56 \cdot 2 / 56 \cdot 6 \\
55 \cdot 4 / 58 \cdot 8 \\
32 \cdot 3 / 38 \cdot 8 \\
37 \cdot 3 / 31 \cdot 2 \\
35 \cdot 2 / 35 \cdot 0 \\
34 \cdot 7 / 42 \cdot 4 \\
30 \cdot 9 / 31 \cdot 7 \\
26 \cdot 4 / 28 \cdot 1 \\
37 \cdot 4 / 34 \cdot 1 \\
25 \cdot 6 / 27 \cdot 3\end{array}$ & $\begin{array}{l}1.40 / 1.31 \\
1.39 / 1.35 \\
1.20 / 1.13 \\
1.76 / 1.71 \\
1.67 / 1.69 \\
1.75 / 1.72 \\
1.40 / 1.42 \\
1.50 / 1.43 \\
1.47 / 1.47 \\
1.46 / 1.46 \\
1.39 / 1.44 \\
1.36 / 1.39 \\
1.47 / 1.44 \\
1.31 / 1.35\end{array}$ & $\begin{array}{l}0.96 / 0.97 \\
0.92 / 0.99 \\
0.97 / 0.96 \\
0.85 / 0.86 \\
0.99 / 0.97 \\
0.90 / 0.97 \\
1.00 / 1.16 \\
0.93 / 0.85 \\
0.93 / 0.92 \\
0.91 / 1.12 \\
0.98 / 0.91 \\
0.87 / 0.86 \\
1.02 / 0.97 \\
0.94 / 0.93\end{array}$ \\
\hline $\begin{array}{l}\text { Mean } \\
\text { SD } \\
\text { CI } \\
\text { pł }\end{array}$ & & $\begin{array}{c}12 \cdot 2 / 12 \cdot 3 \\
3.5 / 3 \cdot 5 \\
10 \cdot 2-14 \cdot 2 / \\
10 \cdot 0-14 \cdot 0 \\
0 \cdot 94\end{array}$ & $\begin{array}{l}11 \cdot 5 / 11 \cdot 7 \\
3 \cdot 6 / 4 \cdot 2 \\
9 \cdot 4-13 \cdot 6 / \\
9 \cdot 2-14 \cdot 2 \\
0 \cdot 92\end{array}$ & & $\begin{array}{c}36 \cdot 7 / 36 \cdot 8 \\
12 \cdot 1 / 12 \cdot 5 \\
29 \cdot 7-43 \cdot 7 / \\
29 \cdot 6-44 \cdot 0 \\
1 \cdot 00\end{array}$ & $\begin{array}{l}1 \cdot 47 / 1 \cdot 45 \\
0 \cdot 16 / 0 \cdot 16 \\
1 \cdot 38-1 \cdot 56 / \\
1 \cdot 36-1.54 \\
0 \cdot 80\end{array}$ & $\begin{array}{l}0.94 / 0.96 \\
0.05 / 0.09 \\
0.91-0.97 / \\
0.91-1.01 \\
0.60\end{array}$ \\
\hline
\end{tabular}

$\mathrm{ND}=$ not done; $\mathrm{CI}=95 \%$ confidence interval.

$\star$ Pubertal stage according to Tanner (reference 12).

tWeight for height ratio according to Jelliffe (reference 16).

$¥$ Cystic fibrosis group $v$ control group. subjects. Standard linear regression analysis was used to relate bone mineral content to age and fat-free mass. An analysis of variance was used to compare the slopes of the regression lines obtained from the two groups. Results are given as mean (SD) and $95 \%$ confidence intervals. A probability value of less than 0.05 was considered significant.

\section{Results}

Cystic fibrosis patients and controls were similar for age, bone age, pubertal stage, weight, height, and weight for height ratio (table 1).

Six cystic fibrosis patients were homozygotes and five were heterozygotes for the mutation F508. One patient had none of the mutations tested for. Controls were not carriers for these mutations. Mean Shwachman-Kulczycki and Crispin-Norman scores of 76 (range 55 to 90) and 9 (range 4 to 24), respectively, suggested that the patients were not severely affected (table 2).

The 72 hour dietary recalls showed that cystic fibrosis patients ingested significantly more energy, protein, calcium, phosphorus, and vitamin $\mathrm{D}$ than controls (table 3 ).

Mean fat-free mass did not differ between cystic fibrosis patients and controls, whether assessed by skinfold thickness [30.9 (SD 10.6) $\mathrm{kg}$ and $31.4(10.8) \mathrm{kg}$, respectively], bioelectrical impedance analysis $[28 \cdot 2(9 \cdot 4) \mathrm{kg}$ and $28.4(9.9) \mathrm{kg}$, respectively], or DEXA [30.2 $(10.7) \mathrm{kg}$ and $29.7(10.8) \mathrm{kg}$, respectively]. Fat-free mass measured by skinfold thickness and DEXA were highly correlated for both cystic fibrosis patients [FFM ${ }_{\text {DEXA }}$ $(\mathrm{kg})=1 \cdot 01^{\star} \mathrm{FFM}_{\text {skinfold }}(\mathrm{kg})-0.96, r^{2}=0.99$, $\mathrm{p}<0.001]$ and controls [FFM DEXA $(\mathrm{kg})=$ $0.95^{\star} \mathrm{FFM}_{\text {skinfold }} \quad(\mathrm{kg})-0.10, \quad r^{2}=0.91$, $\mathrm{p}<0.001$ ]. Fat-free mass measured by bioelectrical impedance analysis gave lower results in both groups by comparison with the two other methods $(p<0.05)$. Fat-free mass values measured by bioelectrical impedance (BIA) and DEXA were also highly correlated in cystic fibrosis patients $\left[\mathrm{FFM}_{\mathrm{DEXA}}\right.$

Table 2 Mutations and disease status in cystic fibrosis (CF) patients

\begin{tabular}{|c|c|c|c|c|}
\hline $\begin{array}{l}C F \\
\text { patients }\end{array}$ & Mutations & & $\begin{array}{l}\text { Shwachman- } \\
\text { Kulczycki } \\
\text { score }\end{array}$ & $\begin{array}{l}\text { Crispin- } \\
\text { Norman } \\
\text { score† }\end{array}$ \\
\hline $\begin{array}{l}1 \\
2\end{array}$ & $\begin{array}{l}\Delta F 508 \\
G 542 x\end{array}$ & $\underset{-\ddagger}{\Delta F} 508$ & $\begin{array}{l}80 \\
75\end{array}$ & $\begin{array}{l}19 \\
12\end{array}$ \\
\hline 3 & $\Delta F 508$ & -+ & $\begin{array}{l}75 \\
70\end{array}$ & $\begin{array}{l}12 \\
11\end{array}$ \\
\hline 4 & $\Delta \mathrm{F} 508$ & G $542 \times$ & 65 & 11 \\
\hline 5 & - & - & 90 & 5 \\
\hline 6 & $\Delta \mathrm{F} 508$ & $\Delta F 508$ & 80 & 4 \\
\hline 7 & $\Delta \mathrm{F} 508$ & $\Delta \mathrm{F} 508$ & 80 & 5 \\
\hline 8 & $\Delta \mathrm{F} 508$ & $\Delta \mathrm{F} 508$ & 85 & 6 \\
\hline 9 & $\Delta \mathrm{F} 508$ & $\Delta \mathrm{F} 508$ & 80 & 9 \\
\hline 10 & $\Delta \mathrm{F} 508$ & $\Delta \mathrm{F} 508$ & 75 & 4 \\
\hline 11 & R $553 \mathrm{X}$ & 3905 ins T & 75 & 9 \\
\hline 12 & $\Delta \mathrm{F} 508$ & 3905 ins T & 55 & 24 \\
\hline 13 & $\Delta \mathrm{F} 508$ & 3905 ins T & 80 & 4 \\
\hline 14 & $\Delta \mathrm{F} 508$ & 3905ins T & 75 & 4 \\
\hline $\begin{array}{l}\text { Mean } \\
\text { SD }\end{array}$ & & & $\begin{array}{r}76 \\
9\end{array}$ & $\begin{array}{l}9 \\
6\end{array}$ \\
\hline
\end{tabular}

* Severity of CF disease according to Shwachman and Kulczycki (reference 9).

†Severity of $x$ ray pulmonary lesions according to Crispin and Norman (reference 10).

$\ddagger$ No usual mutation found. 
Table 372 Hour nutrient intake investigation (cystic fibrosis/control)

\begin{tabular}{llcllc}
\hline Pair & $\begin{array}{l}\text { Energy } \\
(\text { (cal) }\end{array}$ & $\begin{array}{l}\text { Protein } \\
(g)\end{array}$ & $\begin{array}{l}\text { Calcium } \\
(\mathrm{mg})\end{array}$ & $\begin{array}{l}\text { Phosphorus } \\
(\mathrm{mg})\end{array}$ & $\begin{array}{c}\text { Vitamin } D^{\star} \\
(I U)\end{array}$ \\
\hline 1 & $3339 / 2147$ & $132 / 67$ & $1844 / 974$ & $1888 / 1160$ & $1332 / 82$ \\
2 & $2700 / 2220$ & $126 / 60$ & $1668 / 323$ & $1703 / 805$ & $658 / 33$ \\
3 & $2438 / 2593$ & $85 / 81$ & $1695 / 1167$ & $1782 / 1395$ & $982 / 61$ \\
4 & ND/ND & ND/ND & ND/ND & ND/ND & ND/ND \\
5 & $2441 / 1918$ & $84 / 60$ & $887 / 392$ & $1549 / 888$ & $44 / 66$ \\
6 & $3000 / 2883$ & $95 / 80$ & $1469 / 1194$ & $1773 / 1451$ & $1072 / 64$ \\
7 & $2475 / 1446$ & $114 / 54$ & $1244 / 542$ & $1744 / 1055$ & $572 / 35$ \\
8 & $2424 / 1888$ & $95 / 50$ & $1432 / 1063$ & $1580 / 1061$ & $1061 / 77$ \\
9 & $2522 / 2223$ & $89 / 72$ & $1420 / 979$ & $1372 / 1231$ & $1017 / 52$ \\
10 & $2051 / 1991$ & $82 / 69$ & $554 / 390$ & $1538 / 1226$ & $942 / 146$ \\
11 & ND/ND & ND/ND & ND/ND & ND/ND & ND/ND \\
12 & $2120 / 2028$ & $94 / 56$ & $1157 / 754$ & $1317 / 1091$ & $1117 / 50$ \\
13 & $3086 / 1795$ & $116 / 49$ & $2325 / 698$ & $2342 / 765$ & $1007 / 41$ \\
14 & $2566 / 2108$ & $97 / 58$ & $2167 / 813$ & $2235 / 1134$ & $994 / 54$ \\
Mean & $2596 / 2103$ & $101 / 63$ & $1489 / 773$ & $1735 / 1105$ & $900 / 63$ \\
SD & $380 / 370$ & $17 / 11$ & $502 / 310$ & $309 / 212$ & $334 / 30$ \\
CI & $2355-2837 /$ & $90-112 /$ & $1170-1808 /$ & $1539-1931 /$ & $688-1102 /$ \\
& $1868-2338$ & $56-70$ & $576-970$ & $870-1340$ & $44-82$ \\
p† & $<0.01$ & $<0.0001$ & $<0 \cdot 0005$ & $<0.0001$ & $<0.0001$ \\
\hline
\end{tabular}

$\mathrm{ND}=$ not done; $\mathrm{CI}=95 \%$ confidence interval

* For cystic fibrosis patients, from nutritional source and medication.

†Cystic fibrosis group $v$ control group.
Table 4 Bone mineral content (BMC) of subjects (cystic fibrosis/control)

\begin{tabular}{|c|c|c|}
\hline Pair & Whole skeleton $B M C(\mathrm{~kg})$ & Lumbar spine $B M C(\mathrm{~kg})$ \\
\hline $\begin{array}{r}1 \\
2 \\
3 \\
4 \\
5 \\
6 \\
7 \\
8 \\
9 \\
10 \\
11 \\
12 \\
13 \\
14\end{array}$ & $\begin{array}{l}0 \cdot 774 / 0 \cdot 810 \\
0 \cdot 715 / 0 \cdot 864 \\
0 \cdot 568 / 0 \cdot 435 \\
2 \cdot 268 / 2 \cdot 199 \\
2 \cdot 042 / 2 \cdot 192 \\
1 \cdot 954 / 2 \cdot 258 \\
1 \cdot 089 / 1 \cdot 237 \\
1 \cdot 141 / 0 \cdot 894 \\
1 \cdot 123 / 1 \cdot 221 \\
1 \cdot 235 / 1 \cdot 477 \\
0 \cdot 908 / 0 \cdot 972 \\
0 \cdot 692 / 0 \cdot 892 \\
1 \cdot 245 / 1 \cdot 066 \\
0 \cdot 828 / 0 \cdot 764\end{array}$ & $\begin{array}{l}0.019 / 0.019 \\
0.020 / 0.018 \\
0.018 / 0.013 \\
0.061 / 0.059 \\
0.053 / 0.054 \\
0.045 / 0.066 \\
0.025 / 0.022 \\
0.035 / 0.025 \\
0.029 / 0.027 \\
0.029 / 0.030 \\
0.023 / 0.025 \\
0.019 / 0.021 \\
0.030 / 0.029 \\
0.025 / 0.022\end{array}$ \\
\hline $\begin{array}{l}\text { Mean } \\
\text { SD } \\
\text { CI } \\
\mathrm{p}^{\star}\end{array}$ & $\begin{array}{l}1 \cdot 184 / 1 \cdot 229 \\
0 \cdot 536 / 0 \cdot 576 \\
0 \cdot 875-1 \cdot 493 / 0 \cdot 896-1 \cdot 562 \\
0 \cdot 84\end{array}$ & $\begin{array}{l}0.031 / 0 \cdot 031 \\
0.013 / 0 \cdot 016 \\
0.024-0 \cdot 038 / 0 \cdot 022-0.040 \\
0.99\end{array}$ \\
\hline
\end{tabular}

$\mathrm{CI}=95 \%$ confidence interval.

${ }^{\star}$ Cystic fibrosis group $v$ control group.

$(\mathrm{kg})=1 \cdot 13 \star \mathrm{FFM}_{\mathrm{BIA}} \quad(\mathrm{kg}) \quad-1.59, \quad r^{2}=0.99$, $\mathrm{p}<0.001]$ and in controls $\left[\mathrm{FFM}_{\mathrm{DEXA}}(\mathrm{kg})=\right.$ $1.08^{\star}$ fat-free mass BIA $(\mathrm{kg})-1.02, r^{2}=0.99$, $\mathrm{p}<0.001]$. Whole skeleton and lumbar spine bone mineral content were not different from controls in cystic fibrosis patients (table 4). Bone mineral content was highly correlated with fat-free mass (fig 1, data shown only for whole skeleton bone mineral content) and the regression equations were not different among the two groups. Bone mineral content increased

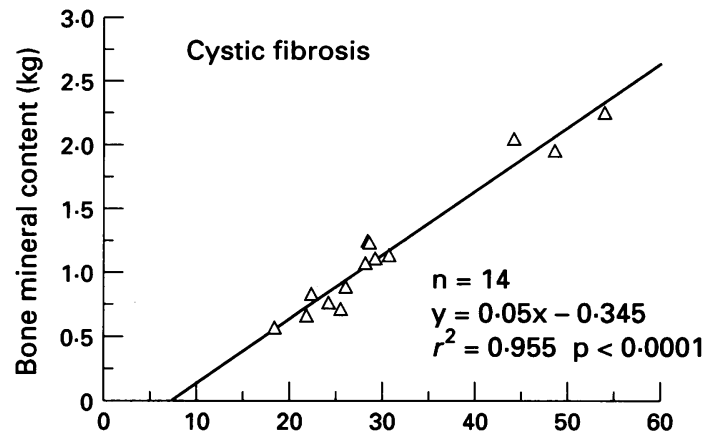

during growth spurt between pubertal stages P1-3 and P4-5 in both groups (fig 2).

Plasma concentrations of calcium, phosphorus, 25-OHD, PTH, and osteocalcin were similar in cystic fibrosis patients and in controls (table 5). Urinary calcium, hydroxyproline, calcium/creatinine ratio, and hydroxyproline/creatinine ratio were also similar (data not shown). Stratification according to gender did not provide more information.

\section{Discussion}

This study shows that bone mineral content of well nourished young cystic fibrosis patients is similar to healthy subjects matched for gender, age, and nutritional status. Our data do not support the suggestion that cystic fibrosis patients may have a primary defect in bone mineral and vitamin $\mathrm{D}$ metabolism.

We follow 21 cystic fibrosis children aged 6 to 20 years old in our outpatient clinic. To avoid a bias of selection, all but one (parental refusal) well nourished patients with a weight to height ratio of $85 \%$ or above were included in the present study. Three patients with protein-energy malnutrition, two with insulin dependent diabetes, and one receiving corticosteroids were not recruited.

Our results have not shown evidence of demineralisation and osteoporosis which has been reported in cystic fibrosis patients. ${ }^{4-6}$ Unfortunately the nutritional status is not well described in these studies. As a whole, our cystic fibrosis patients were not undernourished and had a normal body composition.

Protein-energy malnutrition and growth delay are common features of cystic fibrosis. ${ }^{23} 24$ The major cause is an energy intake which corresponds to $80 \%$ of the US recommended dietary allowance. ${ }^{25}$ The good nutritional status of our cystic fibrosis patients was undoubtedly related to their high protein-energy intake. They ingested about $25 \%$ more energy and about $30 \%$ more protein than controls. Their calcium, phosphorus, and total vitamin $\mathrm{D}$ intake was also much higher, though their dietary vitamin $\mathrm{D}$ intake was very low as there is no vitamin $\mathrm{D}$ fortification of milk in Switzerland. A single 72 hour dietary recall does not reflect nutritional intake over a period of many years. However, our cystic fibrosis patients are seen regularly by dieticians and are encouraged at each visit to

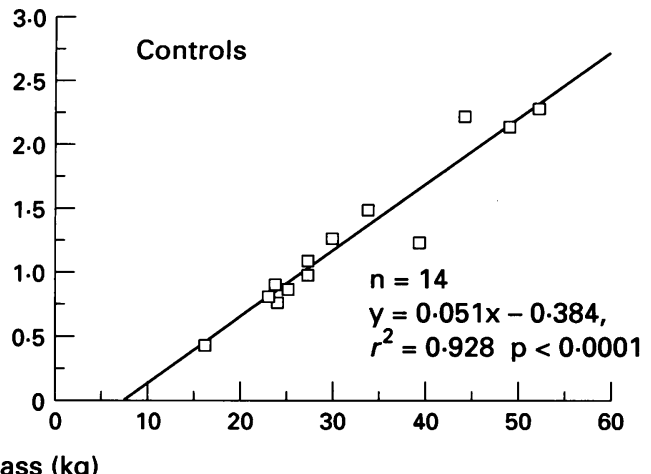

Fat-free mass (kg)

Figure 1 Correlation between bone mineral content and fat-free mass evaluated by anthropometry for cystic fibrosis patients and controls. 


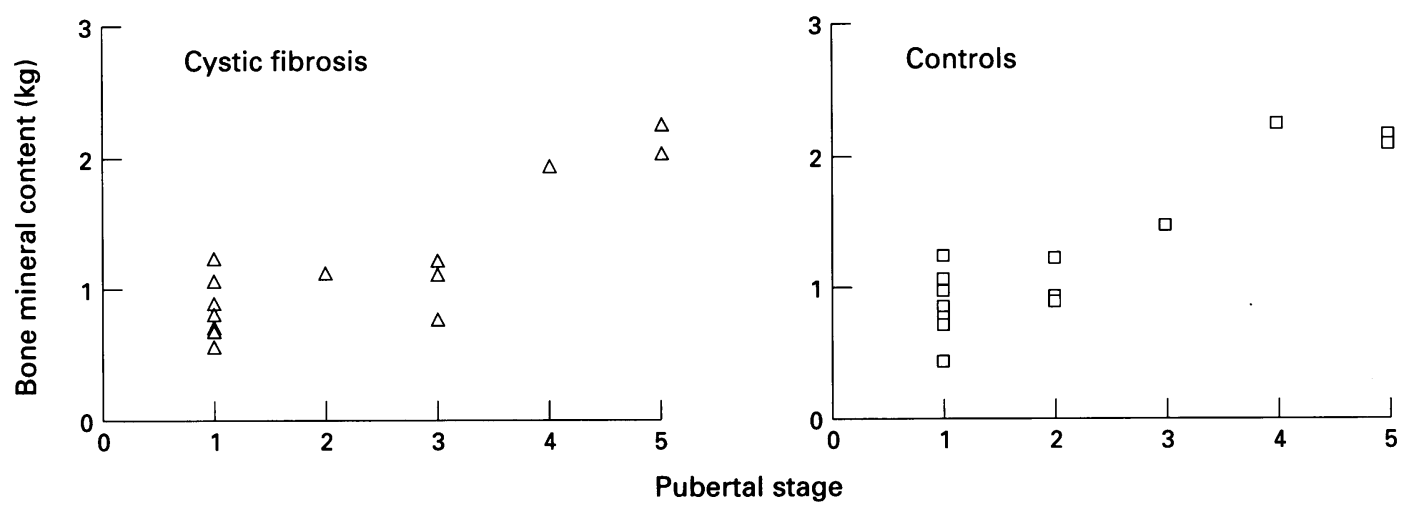

Figure 2 Bone mineral content related to pubertal stages for cystic fibrosis patients and controls.

increase their food intakes. None of our cystic fibrosis patients had manifest clinical malabsorption and all were highly compliant with pancreatic enzyme replacement therapy.

Dual energy $x$ ray absorptiometry is now the gold standard reference method of measuring bone mineral content. Its major advantage is high precision by comparison with carcass analysis $(<1 \%)$, good reproducibility (1\%), and low irradiation (5 mrem).${ }^{26}$ DEXA also allows precise measurement of body composition. ${ }^{21}$ However, in order to correlate bone mineral content with body composition, it is mandatory to use independent methods such as anthropometry and bioelectrical impedance analysis. For an unknown reason, fat-free mass measured by bioelectrical impedance is less than by anthropometry and DEXA. Consequently, we used fat-free mass measured by anthropometry to correlate bone mineral content with body composition. In our study whole skeleton and lumbar bone mineral content of cystic fibrosis patients were not different from those of healthy subjects according to age and pubertal stage. Strong and similar correlations were found between bone mineral content and fatfree mass in both groups.

Bone mineral content increases mainly in the late phases of puberty. ${ }^{27} 28$ Our well nourished cystic fibrosis patients undergo a normal puberty, which in turn results in normal bone mineralisation. Physical activity is known to

Table 5 Laboratory indices of bone mineralisation (cystic fibrosis/control)

\begin{tabular}{|c|c|c|c|c|c|}
\hline Pair & $\begin{array}{l}\text { Calcium } \\
(\text { mmoll })\end{array}$ & $\begin{array}{l}\text { Phosphorus } \\
\text { (mmoll })\end{array}$ & $\begin{array}{l}25 \mathrm{OH} \text {-vitamin D } \\
\text { (nmoll) }\end{array}$ & $\begin{array}{l}P T H \\
(p g / m l)\end{array}$ & $\begin{array}{l}\text { Osteocalcin } \\
(\mathrm{ng} / \mathrm{ml})\end{array}$ \\
\hline $\begin{array}{r}1 \\
2 \\
3 \\
4 \\
5 \\
6 \\
7 \\
8 \\
9 \\
10 \\
11 \\
12 \\
13 \\
14\end{array}$ & $\begin{array}{l}2 \cdot 30 / 2 \cdot 39 \\
2 \cdot 60 / 2 \cdot 37 \\
2 \cdot 36 / 2 \cdot 43 \\
2 \cdot 46 / 2 \cdot 23 \\
2 \cdot 23 / 2 \cdot 21 \\
2 \cdot 40 / 2 \cdot 43 \\
2 \cdot 39 / 2 \cdot 37 \\
2 \cdot 48 / 2 \cdot 36 \\
2 \cdot 40 / 2 \cdot 39 \\
2 \cdot 33 / 2 \cdot 34 \\
2 \cdot 56 / \mathrm{ND} \\
2 \cdot 34 / 2 \cdot 43 \\
2 \cdot 49 / 2 \cdot 44 \\
2 \cdot 47 / 2 \cdot 46\end{array}$ & 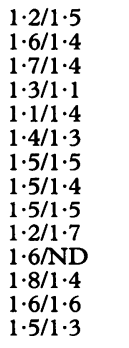 & $\begin{array}{c}73 \cdot 9 / 93 \cdot 6 \\
78 \cdot 1 / 71 \cdot 9 \\
196 \cdot 2 / 153 \cdot 0 \\
70 \cdot 4 / 86 \cdot 4 \\
82 \cdot 4 / 70 \cdot 4 \\
51 \cdot 9 / 107 \cdot 8 \\
18 \cdot 2 / 67 \cdot 9 \\
64 \cdot 4 / 118 \cdot 3 \\
72 \cdot 9 / 116 \cdot 8 \\
92 \cdot 4 / 101 \cdot 3 \\
52 \cdot 4 / \mathrm{ND} \\
41 \cdot 4 / 82 \cdot 9 \\
80 \cdot 9 / 133 \cdot 3 \\
103 \cdot 8 / 130 \cdot 3\end{array}$ & $\begin{array}{l}18 / 29 \\
7 / 20 \\
16 / 8 \\
19 / 28 \\
21 / 23 \\
28 / 12 \\
62 / 30 \\
25 / 7 \\
31 / 27 \\
12 / 39 \\
23 / \mathrm{ND} \\
20 / 24 \\
20 / 15 \\
25 / 31\end{array}$ & $\begin{array}{l}49 \cdot 8 / 82 \cdot 3 \\
53 \cdot 5 / 91 \cdot 6 \\
47 \cdot 8 / 58 \cdot 8 \\
35 \cdot 2 / 23 \cdot 5 \\
44 \cdot 4 / 33 \cdot 8 \\
82 \cdot 1 / 102 \cdot 5 \\
69 \cdot 0 / 82 \cdot 6 \\
94 \cdot 1 / 74 \cdot 9 \\
80 \cdot 1 / 76 \cdot 3 \\
92 \cdot 9 / 142 \cdot 5 \\
64 \cdot 1 / \mathrm{ND} \\
81 \cdot 9 / 108 \\
92 \cdot 6 / 106 \cdot 2 \\
84 \cdot 0 / 90 \cdot 3\end{array}$ \\
\hline $\begin{array}{l}\text { Mean } \\
\text { SD } \\
\text { CI } \\
p^{\star}\end{array}$ & $\begin{array}{l}2 \cdot 42 / 2 \cdot 37 \\
0 \cdot 10 / 0 \cdot 08 \\
2 \cdot 36-2 \cdot 48 / \\
2 \cdot 32-2 \cdot 42 \\
0 \cdot 24\end{array}$ & $\begin{array}{l}1 \cdot 5 / 1 \cdot 4 \\
0 \cdot 2 / 0 \cdot 1 \\
1 \cdot 3-1 \cdot 6 / \\
1 \cdot 3-1 \cdot 5 \\
0 \cdot 55\end{array}$ & $\begin{array}{l}77 \cdot 1 / 102 \cdot 6 \\
40 \cdot 6 / 26 \cdot 8 \\
53 \cdot 7-100 \cdot 5 / \\
86 \cdot 4-118 \cdot 8 \\
0 \cdot 67\end{array}$ & $\begin{array}{c}23 / 22 \\
13 / 10 \\
15-31 / \\
16-28 \\
0 \cdot 85\end{array}$ & $\begin{array}{l}69 \cdot 4 / 82 \cdot 6 \\
20 \cdot 1 / 31 \cdot 4 \\
57 \cdot 8-81 \cdot 0 / \\
63 \cdot 6-101 \cdot 6 \\
0 \cdot 20\end{array}$ \\
\hline
\end{tabular}

$\mathrm{PTH}=$ parathyroid hormone; $\mathrm{ND}=$ not done; $\mathrm{CI}=95 \%$ confidence interval. $\star$ Cystic fibrosis group $v$ control group. have a positive influence on lumbar mineralisation. ${ }^{29}$ In a recent study, we have shown that our cystic fibrosis population has only slightly reduced spontaneous physical activity. ${ }^{30}$ Serum 25-OHD depends on sunlight exposure and for this reason we measured the patients and their controls at the same time. The lowest value of serum $25-\mathrm{OHD}$ was measured in cystic fibrosis patient number 7 in December. This may have been the result of faster depletion of reserves stocked in summer in cystic fibrosis patients than in controls. ${ }^{31}{ }^{32}$ No secondary hyperparathyroidism was observed, except perhaps in patient number 7 . The protein turnover of bone seems to be similar in cystic fibrosis patients and controls, as suggested by identical levels of osteocalcin.

From our data we conclude that cystic fibrosis patients probably have no primary defect in bone mineral and vitamin $\mathrm{D}$ metabolism, and consequently a normal bone mineral content when they are not malnourished. A bone mass deficit in a particular cystic fibrosis patient reflects general malnutrition, as reported recently in adults. ${ }^{33} \mathrm{~A}$ larger population has to be studied for a definitive answer.

We thank Dr Francine Thonney for genetic screening; Dr Claude Roy for advices and manuscript corrections; Nicoletta Claude Roy for advices and manuscript corrections; Nicolett Jacquet for special laboratory analysis; Françoise Dubied for Jacquet for special laboratory analysis; Françoise Dubied for technical assistance; Philippe Frascarolo for statistical advice; and radiologic technologists for DEXA examination. This
was supported by the Swiss Society for Cystic Fibrosis.

1 FitzSimmons SC. The changing epidemiology of cystic fibrosis. F Pediatr 1993; 122: 1-9.

2 Gaskin KJ, Waters DLM, Soutter VL, et al. Body composition in cystic fibrosis. Basic Life Sci 1990; 55: 15-21.

3 Newby MJ, Keim NL, Brown DL. Body composition of adult cystic fibrosis patients and control subjects as determined by densitometry, bioelectrical impedance, total-body electrical conductivity, skinfold measurements, and deuterium oxide dilution. Am f Clin Nutr 1990; 52: 209-13.

4 Mischler EH, Chesney PJ, Chesney RW, Mazess RB. Demineralization in cystic fibrosis detected by direct Demineralization in cystic fibrosis detected by direct
photon absorptiometry. Am $\mathcal{f}$ Dis Child $1979 ; 133: 632-5$.

5 Gibbens DT, Gilsanz V, Boechat MI, Dufer D, Carlson ME, Wang CI. Osteoporosis in cystic fibrosis. $\mathcal{F}$ Pediat 1988; 113: 295-300.

6 Bachrach LK, Loutit CW, Moss RB. Osteopenia in adults with cystic fibrosis. Am $\mathcal{F}$ Med 1994; 96: 27-34

7 Bonjour JP, Theintz G, Buchs B, Slosman D, Rizzoli R Critical years and stages of puberty for spinal and femoral bone mass accumulation during adolescence. $\mathcal{f}$ Clin Endocrinol Metab 1991; 73: 555-63.

8 Gilsanz V, Gibbens DT, Roe TF, et al. Vertebral bone density in children: effect of puberty. Radiology 1988; 166: 847-50.

9 Burckhardt P, Michel C. The peak bone mass concept. Clin Rheumatol 1989; 8: 16-21.

10 Shwachman H, Kulczycki LL. Long-term study of one hundred five patients with cystic fibrosis. Am 7 Dis Child 1958; 96: 6-15.

11 Chrispin AR, Norman AP. The systematic evaluation of the chest radiograph in cystic fibrosis. Pediatr Radiol 1974; 2: 101-6. 
12 Tanner JM. Growth at adolescence. Oxford: Blackwell Scientific Publications, 1969.

13 Krause MV, Mahan LK. Food, nutrition and diet therapy. Philadelphia: W B Saunders, 1979: 220-31.

14 Souci SW, Fachmann W, Kraut H. Food composition and nutrition tables 1989/90. Stuttgart: Wissenschaftliche Verlagsgesellschaft mbH, 1989/90.

15 Klepping J, Guilland JC, Fuchs F, Marcer I, HouardMalval M. Recueil de données sur la composition des aliments. Laboratoire de Physiologie de l'UFR de Médecine de l'Université de Physiologie de l'UFR de Medecine de l'Universite de Dijon. Centre d'Etude et d'Information
sur les Vitamines. Neuilly-sur Seine Cedex: Produits sur les

16 Jelliffe DB. The assessment of the nutritional status of the community. Geneva: World Health Organisation, 1966.

17 Deurenberg P, Pieters JJ, Hautvast JGAJ. The assessment of the body fat percentage by skinfold thickness measurements in childhood and young adolescence. $\mathrm{Br} \mathcal{F}$ Nutr 1990; 63: 293-303.

18 Spicher V, Roulet M, Schaffner C, Schutz Y. Bio-electrical impedance analysis for estimation of fat-free mass and muscle mass in cystic fibrosis patients. Eur 7 Pediatr 1993 152: 222-5.

19 Lukaski HC, Johnson PE, Bolonchuk WW, Lykken GI. Assessment of fat-free mass using bioelectrical impedance measurements of the human body. Am $\mathcal{F}$ Clin Nutr 1985; 41: 810-7.

20 Deurenberg $\mathrm{P}$, van der Kooy K, Leenen R, Weststrate JA, Seidell JC. Sex and age specific prediction formulas for estimating body composition from bioelectrical impedance: a cross-validation study. Int $f$ Obes $1991 ; 15: 17-25$.
Mazess RB, Barden HS, Bisek JP, Hanson J. Dual-energy xray absorptiometry for total-body and regional bonemineral and soft-tissue composition. Am f Clin Nutr 1990; 51: 1106-12.

22 Greulich WW, Pyle SI. Radiographic atlas of skeletal development of the hand and wrist. Stanford, California: Stanford University Press, 1976.

23 Parsons HG, Beaudry P, Dumas A, Pencharz PB. Energy needs and growth in children with cystic fibrosis. $f$ Pediatr Gastroenterol Nutr 1983; 2: 44-9.

24 Kraemer R, Rüdeberg A, Hadorn B, Rossi E. Relative underweight in cystic fibrosis and its prognostic value. Acta Paediatr Scand 1978; 67: 33-7.

25 Chase HP, Long MA, Lavin MH. Cystic fibrosis and malnutrition. F Pediatr 1979; 95: 337-47.

26 Chan GM. Performance of dual-energy $x$-ray absorptiometry in evaluating bone, lean body mass, and fat in pediatric try in evaluating bone, lean body mass, and fat

27 Dhuper S, Warren MP, Brooks-Gunn J, Fox R. Effects of hormonal status on bone density in adolescent girls. $\mathcal{f}$ Clin Endocrinol Metab 1990; 71: 1083-8.

28 Riis BJ, Krabbe S, Christiansen C, Catherwood BD, Deftos LJ. Bone turnover in male puberty: a longitudinal study. Calcif Tissue Int 1985; 37: 213-7.

29 Slemenda CW, Miller JZ, Hui SL, Reister TK, Johnston CC. Role of physical activity in the development of skeletal mass in children. 7 Bone Miner Res 1991; 6: 1227-33.

30 Spicher V, Roulet M, Schutz Y. Assessment of total energy expenditure in free-living patients with cystic fibrosis. $\mathcal{f}$ Pediatr 1991; 118: 865-72.

31 Reiter EO, Brugman SM, Pike JW, et al. Vitamin D metabolites in adolescents and young adults with cystic fibrosis: lites in adolescents and young adults with cystic fibrosicts of sun and season. $₹$ Pediatr 1985; 106: $21-6$.

32 Weisman Y, Reiter E, Stern RC, Root A. Serum concentrations of 25-hydroxyvitamin D and 24,25-dihydroxyvitamin D in patients with cystic fibrosis. $\mathcal{F}$ Pediatr 1979; 95: $416-8$

33 Rochat T, Slosman DO, Pichard C, Belli DC. Body composition analysis by dual-energy X-ray absorptiometry in adults with cystic fibrosis. Chest 1994; 106: 800-5. 